\title{
FishMap Zv8 Update-A Genomic Regulatory Map of Zebrafish
}

\author{
Deeksha Bhartiya, Jayant Maini, Meenakshi Sharma, Prateek Joshi, Saurabh V. Laddha, \\ Saakshi Jalali, Ashok Patowary, Ramya Purkanti, Mukesh Lalwani, Angom Ramcharan Singh, \\ Rajendra Chauhan, Naresh Singh, Anshu Bhardwaj, Vinod Scaria, and Sridhar Sivasubbu
}

\begin{abstract}
The advancements in genomics technologies and the amenability to large-scale computational analysis have contributed immensely to the understanding of the zebrafish genome, its organization, and its functional correlates. Translating genomics information into biological meaning would require integration and amenability of data and tools. FishMap is a community resource for genomic datasets on zebrafish created with a vision to provide relevant and readily available information to zebrafish researchers. The present update of FishMap has kept up with the availability of the latest zebrafish genome assembly (Zv8). In this update, particular emphasis has been given to noncoding RNAs and noncoding RNA-mediated regulation in addition to genomic regulatory motifs, which are emerging areas of vertebrate biology. FishMap Zv8 update also features a sequence mapping and analysis server. Consistent with its commitment to make the information freely available to the community, FishMap features options to share data between compatible resources in addition to making it amenable to programmatic access. FishMap Zv8 update is available at http://fishmap2.igib.res.in.
\end{abstract}

\section{Introduction}

$\mathbf{P}$ Reviously, we PUblished FishMap ${ }^{1}$ as a unified and centralized community resource for integration, visualization, and analyses of postgenomics datasets on zebrafish, based on the Zv7 build of the zebrafish genome. With the latest zebrafish genome build (Zv8) now being available, it was imperative to create an updated version of the resource. In this update, a balanced blend of datasets were chosen. While keeping general tracks on gene annotation, emphasis was given to emerging research areas in vertebrate biology, especially in the areas of genome regulation, including noncoding RNAs (ncRNAs) and ncRNA-mediated regulation. The datasets incorporated in the current update encompass the diversity of research in zebrafish genomics ranging from high-throughput experimental data to genome-scale computational predictions and analysis.

ncRNA and ncRNA-mediated regulation have attracted immense scientific interest. ${ }^{2}$ MicroRNAs, a major class of ncRNAs, and their regulation have been one of the areas of active research. $^{3-5}$ In the new update of FishMap, emphasis has been given to the new field of ncRNAs and ncRNAmediated regulation. MicroRNAs from the latest version (v13) of miRbase database (http://microrna.sanger.ac.uk/) and predicted microRNA targets have been incorporated. In addition to transfer RNAs predicted using tRNAscan-SE, a number of computationally predicted ncRNAs have been included in the newer release (see Supplemental Tables S1 and S2; available online at www.liebertonline.com). We have used Erpin, ${ }^{6}$ one of the most popular ncRNA analysis methods for genome-wide prediction of potential ncRNA motifs in the zebrafish genome. These include genome-wide predictions of a number of microRNA families and small nucleolar RNAs, in addition to other regulatory RNA motifs.

Regulation of ncRNAs have traditionally been achieved by exogenously provided antisense oligonucleotides, usually with backbone modifications such as locked nucleic acids, morpholinos, ${ }^{8}$ and DNAzymes. ${ }^{9}$ Recently, antagomi Rzymes ${ }^{10}$ have also been successfully used to downregulate microRNAs. The present update of FishMap features predesigned antagomiRzyme sequences along with the appropriate target microRNA sequences as a ready resource for researchers to select them for appropriate experiments.

FishMap update also includes data on computational prediction of regulatory motifs in the zebrafish genome. G-quadruplex motifs have been recently shown to have a role in gene regulation, both in expression of the genes as well as at the transcript level. ${ }^{11-13}$ FishMap Zv8 update encompasses

Institute of Genomics and Integrative Biology (Council of Scientific and Industrial Research), Delhi, India. 
genome-wide analysis of G-quadruplex motifs using the QuadFinder algorithm, ${ }^{14}$ thus serving a ready resource for researchers working in this field. In addition to the genomewide nucleosome exclusion signal predictions, ${ }^{15}$ a number of predicted $3^{\prime}$ and $5^{\prime}$ untranslated motif information have been incorporated for those interested in studying regulatory modules in vertebrate gene regulation.

Keeping in mind the increasingly popular next-generation sequencing technology for transcriptome analysis, we have included a separate track on Digital Gene Expression tags. ${ }^{16}$ Further, a number of new tracks relating to Pathways, Gene Ontology Annotations, Protein motifs, Variations, and Gene regulation have been incorporated in this update. In addition to the data updates, FishMap Zv8 features improved functionalities such as BLAT and TBLASTN servers for mapping and analyzing custom sequences on the genome. Further, FishMap Zv8 permits sharing of data tracks across similar databases and supports data interoperability through community standards.

\section{Future Directions}

FishMap is committed to serve the zebrafish genomics community with relevant and up-to-date resource on zebrafish genomics. The resource will be constantly updated, keeping pace with the latest developments in zebrafish genomics. In the near future, the database will expand to encompass the diversity and scale of genomic information that is being generated from high-throughput sequencing and transcriptomics technologies. This may also include recent wholegenome resequencing initiatives and genome-scale activities to understand the transcriptome and variome. Consistent with our commitment to making the data freely sharable and interoperable with other databases and resources, FishMap is poised to be one of the major complementary resource for zebrafish community annotation initiatives.

\section{Acknowledgments}

The research was supported by a grant (FAC002) from the Council of Scientific and Industrial Research, India. The authors thank members of the zebrafish community for freely sharing their data.

\section{Disclosure Statement}

No competing financial interests exist.

\section{References}

1. Meli R, et al. FishMap: a community resource for zebrafish genomics. Zebrafish 2008;5:125-130.

2. Mattick JS. Non-coding RNAs: the architects of eukaryotic complexity. EMBO Rep 2001;2:986-991.

3. Begemann G. MicroRNAs and RNA interference in zebrafish development. Zebrafish 2008;5:111-119.

4. Giraldez AJ, et al. Zebrafish MiR-430 promotes deadenylation and clearance of maternal mRNAs. Science 2006; 312:75-79.
5. Giraldez AJ, et al. MicroRNAs regulate brain morphogenesis in zebrafish. Science 2005;308:833-838.

6. Lambert A, et al. The ERPIN server: an interface to profilebased RNA motif identification. Nucleic Acids Res 2004;32: W160-W165.

7. Kauppinen S, Vester B, Wengel J. Locked nucleic acid: highaffinity targeting of complementary RNA for RNomics. Handb Exp Pharmacol 2006;173:405-422.

8. Moulton JD, Yan YL. Using Morpholinos to control gene expression. Curr Protoc Mol Biol 2008;Chapter 26, Unit 26.8.

9. Achenbach JC, Chiuman W, Cruz RP, Li Y. DNAzymes: from creation in vitro to application in vivo. Curr Pharm Biotechnol 2004;5:321-336.

10. Jadhav VM, Scaria V, Maiti S. Antagomirzymes: oligonucleotide enzymes that specifically silence microRNA function. Angew Chem Int Ed Engl 2009;48:2557-2560.

11. Burge S, Parkinson GN, Hazel P, Todd AK, Neidle S. Quadruplex DNA: sequence, topology and structure. cleic Acids Res 2006;34:5402-5415.

12. Arora $\mathrm{A}$, et al. Inhibition of translation in living eukaryotic cells by an RNA G-quadruplex motif. RNA 2008;14:12901296.

13. Wong HM, Payet L, Huppert JL. Function and targeting of G-quadruplexes. Curr Opin Mol Ther 2009;11:146-155.

14. Scaria V, Hariharan M, Arora A, Maiti S. Quadfinder: server for identification and analysis of quadruplex-forming motifs in nucleotide sequences. Nucleic Acids Res 2006;34:W683W685.

15. Luykx P, Bajic IV, Khuri S. NXSensor web tool for evaluating DNA for nucleosome exclusion sequences and accessibility to binding factors. Nucleic Acids Res 2006;34: W560-W565.

16. Reinartz J, et al. Massively parallel signature sequencing (MPSS) as a tool for in-depth quantitative gene expression profiling in all organisms. Brief Funct Genomics Proteomics 2002;1:95-104.
Address correspondence to: Vinod Scaria, MBBS Institute of Genomics and Integrative Biology (Council of Scientific and Industrial Research) Mall Road Delhi 110007 India

E-mail: vinods@igib.in

Sridhar Sivasubbu, Ph.D. Institute of Genomics and Integrative Biology (Council of Scientific and Industrial Research) Mall Road Delhi 110007 India

E-mail: sridhar@igib.in 